CASE REPORT

\title{
Atrial fibrillation after vardenafil therapy
}

H H Veloso, A A V de Paola

Emerg Med J 2005;22:823. doi: 10.1136/emj.2004.015982

Vardenafil is a new oral phosphodiesterase inhibitor used for erectile dysfunction. We report a case admitted with a firstdetected, symptomatic paroxysmal atrial fibrillation in a healthy patient after self-medication with vardenafil.

\section{CASE REPORT}

Vardenafil (Levitra ${ }^{\circledR}$ ) is the second oral phosphodiesterase inhibitor approved for erectile dysfunction. ${ }^{12}$ As well as sildenafil (Viagra ${ }^{\circledR}$ ), this drug acts by relaxing blood vessels and muscles in the penis, allowing increased blood flow into the penis to produce an erection. The most important hazard adverse effect related with this therapy is the promotion of a profound hypotension and myocardial ischemia when combined with nitrates. Cases of atrial fibrillation had been related with sildenafil ${ }^{3}$ but, with vardenafil therapy, this arrhythmia was not described yet. We report a case with a first-detected, symptomatic paroxysmal atrial fibrillation in a healthy patient after a self-medication with vardenafil.

A 50 year old man presented to the emergency room with a persistent palpitation, lasting for 2 hours, began 15 minutes after ingesting, as self-medication, a single $10 \mathrm{mg}$ tablet of vardenafil. He denied other symptoms or the use of ethanol or other medicines. He had no significant history for erectile dysfunction, and justified the self-medication to improve his sexual performance. He had already used sildenafil previously without complication. He had no cardiovascular antecedents.

At physical examination, an irregular and tachycardic heart rhythm was evidenced, around 140 beats/minute, with no other pathological findings. The electrocardiogram revealed atrial fibrillation with rapid ventricular rate and was without ST segment or $\mathrm{T}$ wave abnormalities. Chest radiograph and routine laboratories including cardiac enzymes were normal. Beyond rest, he was medicated with oral diazepam, and the arrhythmia coursed with conversion to normal sinus rhythm after four hours. He obtained hospital discharge without antiarrhythmic medication.

It was performed on outpatient cardiovascular investigation. The transthoracic Doppler echocardiography disclosed a structurally normal heart, with left atrial diameter of $2.8 \mathrm{~cm}$ and left ventricular ejection fraction of 0.76 . The 24 hour ambulatory electrocardiographic monitoring did not present cardiac arrhythmias, and the treadmill exercise testing was negative for myocardial ischemia. After a follow-up of 7 months, the patient was asymptomatic, without medication.

\section{DISCUSSION}

The temporal relationship between the ingestion of vardenafil and new onset atrial fibrillation in a patient with no cardiovascular diseases suggests that the drug caused the

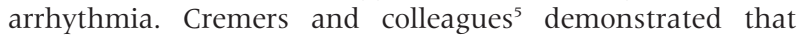
sildenafil has a potent vasodilatory action but has no direct influence on human myocardial contractility or proarrhythmic effects in vitro. Thus, a possible mechanism involved with the induction of atrial fibrillation is hypotension leading to a reflex tachycardia through catecholamine excess.

Han and Hoffman ${ }^{3}$ reported a case with atrial fibrillation in a patient with normal heart after taking sildenafil. In this case, the patient presented after a syncopal episode, and antiarrhythmic therapy with ibutilide failed to restore normal heart rhythm, which was spontaneously obtained 2 days later. In the other report of atrial fibrillation after sildenafil therapy, Awan et al treated a patient with hypertrophic obstructive cardiomyopathy.

In our case, antiarrhythmic treatment was not necessary to restore normal sinus rhythm as well as for the prevention of recurrences. We believe that patients who have a clear trigger for atrial fibrillation should not undergo antiarrhythmic therapy to restore normal sinus rhythm if the episode is well tolerated. In such cases, the initiation of rate control drugs might be the best approach.

This report strengthens the risk of self-medication with phosphodiesterase inhibitors in a patient without significant erectile dysfunction. This therapy should be restricted for patients with organic urologic dysfunctions and not as a recreational drug.

\section{Authors' affiliations}

H H Veloso, A A V de Paola, VOTCOR - Hospital da Venerável Ordem Terceira da Penitência, Rio de Janeiro, Brazil

Correspondence to: Dr. Henrique Horta Veloso, VOTCOR - Hospital da Venerável Ordem Terceira da Penitência, Rua Conde de Bonfim 1033, Tijuca, Rio de Janeiro - RJ, Brazil, ZIP: 20530-001; hhorta@cardiol.br

Accepted for publication 27 April 2004

\section{REFERENCES}

1 FDA approves new drug for treatment of erectile dysfunction in men. FDA Consum 2003;37(5):7.

2 Crowe SM, Streetman DS. Vardenafil treatment for erectile dysfunction. Ann Pharmacother 2004;38:77-85.

3 Hahn IH, Hoffman RS. Aroused to atrial fibrillation? Am J Emerg Med 2000; 18:642.

4 Awan GM, Calderon E, Dawood G, et al. Acute, symptomatic atrial fibrillation after sildenafil citrate therapy in a patient with hypertrophic obstructive cardiomyopathy. Am J Med Sci 2000;320:69-71.

5 Cremers B, Scheler M, Maack C, et al. Effects of sildenafil (viagra) on human myocardial contractility, in vitro arrhythmias, and tension of internal mammaria arteries and saphenous veins. J Cardiovasc Pharmacol $2003 ; 41: 734-43$ 


\section{"My back is killing $\mathrm{me}^{\prime}$ F Garnham, P Longstaff}

This is a case of a rare cause of back pain that presented to several clinicians before the diagnosis became obvious and the correct management was initiated. Lemierre's syndrome was confirmed at post mortem examination. We conclude that thoracic back pain should not be assumed to have a simple mechanical cause and that efforts should be made to discover the underlying aetiology.

A 30 year old white woman presented to a GP complaining of thoracic back pain. The pain had been present from waking the previous day and had not resolved with ibuprofen. She had recently been coryzal but had no other past medical history of note. On examination, the GP found bilateral paravertebral muscle spasm in the mid to lower thoracic region, for which she was prescribed diclofenac.

The following day the patient presented to the Chelsea and Westminster Hospital, complaining that her back pain had worsened. She reported that it was exacerbated by movement and deep inspiration. Further history revealed that she had suffered from a severe sore throat and a dry cough accompanied by sweating a week previously. On examination, it was noted that she had difficulty standing and was tender adjacent to L2. A neurological examination was performed, which was normal, and she was discharged with diazepam and paracetamol. The respiratory system and neck were not examined, no baseline observations were recorded, and no investigations were performed.

The next morning the patient was seen by a physiotherapist who felt the pain was not mechanical and referred her back to the accident and emergency (A\&E) department. As she booked back into A\&E she appeared pale, so was put on a trolley to lie down. She immediately felt much better, her colour returned to normal, and she returned to a chair in the minor injury waiting room. She declined analgesia, was triaged as standard, and waited to be seen by a doctor. Approximately 5 hours later (22.50) it was noticed that she had become pale and uncomfortable. The first set of observations were performed (temperature $37^{\circ} \mathrm{C}$, heart rate (HR) 132 beats/min, blood pressure (BP) 96/58 mmHg, respiration rate (RR) 20 breaths/min, oxygen saturation $85 \%$ on air), the emergency medicine middle grade and senior house officer were called, and the patient was moved into the resuscitation room.

Table 1 Arterial blood gases

\begin{tabular}{lcc}
\hline & $\begin{array}{l}\text { On air, } \\
\text { at } 23.07\end{array}$ & $\begin{array}{l}\text { On } 60 \% \\
\mathbf{O}_{2} \text { at } 01.13\end{array}$ \\
\hline $\mathrm{pH}$ & 7.298 & 7.17 \\
$\mathrm{pCO}_{2}(\mathrm{kPa})$ & 5.49 & 6.77 \\
$\mathrm{pO}_{2}(\mathrm{kPa})$ & 5.75 & $10.73 \mathrm{a}$ \\
$\mathrm{HCO}_{3}(\mathrm{mmol} / \mathrm{l})$ & 18.9 & 16.3 \\
$\mathrm{BE}(\mathrm{mmol} / \mathrm{l})$ & -6.7 & -10.3 \\
\hline
\end{tabular}

Further history revealed that the patient had developed nausea, diarrhoea, and a cough productive of green sputum overnight, and she had been successfully treated for cervical malignancy 10 years previously.

On examination, there was a clear airway, with nothing abnormal detected in the oropharynx/neck. High flow oxygen was applied via a Hudson mask. RR was 26 breaths/min, trachea central, and percussion was dull at both bases. There was reduced air entry bilaterally with crepitations throughout both lungs and oxygen saturation of $97 \%$ on 15 litres of oxygen. HR was 132 beats/min, BP $96 / 58 \mathrm{mmHg}$, jugular venous pressure unseen, and she had normal heart sounds. An intravenous infusion of $0.9 \%$ saline was started. The patient was drowsy but has Glasgow Coma Score of 5/15, blood glucose $5.5 \mathrm{mmol} / \mathrm{l}$. The abdomen was distended but soft, with mild tenderness centrally but no signs of peritonism. There was bilateral leg swelling to the knees with a slightly tender left calf, which felt hot and tense. There was no rash and otherwise the examination was normal.

A working diagnosis of pneumonia was made and antibiotics (cefuroxime and erythromycin) were immediately administered.

ECG showed sinus tachycardia. Arterial blood gases are shown in table 1 . Chest $x$ ray showed a left sided pleural effusion, multifocal consolidation (most marked in the lingula and right base), and patchy infiltrate in the right upper lobe, and the left hilum appeared enlarged (fig 1). Full blood count showed haemoglobin $134 \mathrm{~g} / \mathrm{l}$, white blood cells $9 \times 10^{9} / \mathrm{l}$, platelets $34 \times 10^{9} / \mathrm{l}$. Urine and electrolyte analysis showed sodium $33 \mathrm{mmol} / \mathrm{l}$, potassium $3.7 \mathrm{mmol} / \mathrm{l}$, urea $33.3 \mathrm{mmol} / \mathrm{l}$, and creatinine $432 \mu \mathrm{mol} / \mathrm{l}$, while liver function tests were: bilirubin $31 \mu \mathrm{mol} / \mathrm{l}$, albumin $20 \mathrm{~g} / \mathrm{l}$, otherwise normal. Prothrombin time was 13.4 seconds, activated partial thromboplastin time 354 seconds, thrombin time 13 seconds, and urine was positive for blood and protein.

The patient was reviewed by the medical team and transferred directly to the intensive thereapy unit. Despite maximum therapy with crystalloids, colloids, and platelets, she continued to deteriorate. At 04.30 she was intubated and ventilated, and inotropes were started. Unfortunately, she died later that morning at 10.38 .

Blood cultures later grew Fusobacterium necrophorum. Postmortem revealed fibrinopurulent exudates in the pleural cavities and multiple necrotic areas. Microscopy revealed numerous microabscesses. There was no evidence of deep venous thrombosis or pulmonary thromboembolism. A diagnosis of Lemierre's syndrome was made.

\section{DISCUSSION}

Lemierre was a Professor of Microbiology in Paris who described a group of 20 patients with very similar symptoms to our patient, who developed "postanginal sepsis". ${ }^{1}$ The group consisted of young adults who had suffered from an infection that spread to cause local thrombophlebitis and distant septic emboli. The main causative organism was $F$ necrophorum.

Abbreviations: A\&E, accident and emergency; BP, blood pressure; $H R$, heart rate; $R R$, respiration rate 


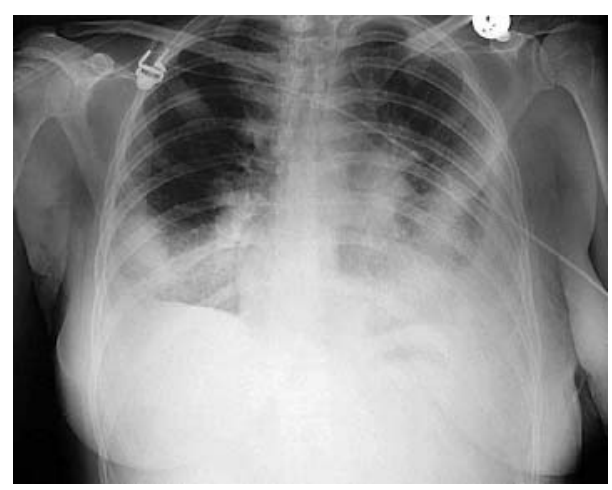

Figure 1 Chest $x$ ray showing left sided pleural effusion, multifocal consolidation, patchy infiltrate in right upper lobe and enlarged left hilum.

$F$ necrophorum is a gram negative, non-spore forming, anaerobic bacillus that occasionally proliferates in a cavity to produce local infection followed by distant spread via the blood (infection with anaerobic streptococci or other fusobacterium can cause a similar picture). ${ }^{2}$ The most usual history is of infection of the tonsils or peritonsillar region, which spreads to the nearby veins causing a thrombophlebitis (patients often complain of pain along the sternocleidomastoid because of involvement of the internal jugular vein). Approximately $7-15$ days later, they develop a febrile illness and symptoms of septic spread. The most commonly affected organs are the lungs (85\%), but joints $(26 \%)$, the peritoneum, liver, kidney, brain, bones, heart, and meninges (more common if the primary focus is otitis media) can all be involved..$^{2-4}$ There have also been some reports of the presenting complaint being a gluteal or psoas abscess or a picture of haemolytic uraemic syndrome. ${ }^{5}$ Less commonly, the problem can originate from otitis media, mastoiditis, purulent endometritis, appendicitis (especially gangrenous), or infections of the urinary tract.

Many patients $(50-75 \%)^{124}$ have a raised bilirubin at presentation, often with hepatomegaly. The white blood cell count is usually in the range of $13-30 \times 10^{9} / 1$ and red blood cell count is often low with a haemolytic picture.

$F$ necrophorium can be isolated from blood cultures in 2-4 days, which in a condition that has a mortality rate of $>80 \%^{2}$ if left untreated may cause a problem. The most recent microbiology paper that we found was a retrospective analysis of all reports of $F$ necrophorium bacteraemia received by the UK Communicable Disease Surveillance Centre between 1990 and 2000. ${ }^{6}$ There were 208 reported cases in England and Wales over the 10 year period with a peak incidence in late winter and a peak age range of 16-23 years. All isolates were susceptible to metronidazole, 15\% were resistant to erythromycin, and $2 \%$ were resistant to penicillin.

Treatment consists of antibiotics and is supportive in most cases (reducing mortality to approximately 15\%), with ligation of the internal jugular vein only needed very rarely in cases with recurrent septic emboli. ${ }^{2}$

There are several important lessons to be learnt from this case. Patients who return with the same complaint should be seen promptly by a senior doctor, and a safety mechanism should be in place for occasions when the senior doctors are unexpectedly busy (on this occasion with patients in the resuscitation room). Any patient who appears unwell at any time during their attendance should have their observations promptly performed/repeated. It also demonstrates the problems encountered in diagnosing Lemierre's syndrome, and that failure to diagnose the syndrome in its early phase may lead to a fatal outcome. Lastly, we believe this case highlights the important message that thoracic back pain often has an underlying aetiology, other than a simple mechanical cause, which should be actively sought with a thorough history, examination, and relevant investigations.

\section{Authors' affiliations \\ F Garnham, P Longstaff, Chelsea and Westminster Hospital, London, UK}

Competing interests: none declared

Correspondence to: Dr F Garnham, Chelsea and Westminster Hospital, London SW10 9NH, UK; francesca.garnham@chelwest.nhs.uk

Accepted for publication 8 March 2004

\section{REFERENCES}

1 Lemierre A. On certain septicaemias due to anaerobic organisms. Lancet 1936;1:701-3.

2 Barker J, Winer-Muram HT, Grey SW. Lemierre syndrome. South Med J 1996;89:1021-3.

3 Abele-Horn M, Emmerling P, Mann JF. Lemierre's syndrome with spondylitis and pulmonary and gluteal abscesses associated with Mycoplasma pneumoniae pneumonia. Eur J Clin Microbiol Infect Dis 2001;20:263-6.

4 Hagelskjaer K, Prag J. Lemierre's syndrome. Clin Infect Dis 2000;31:524-32.

5 Chand DH, Brady RC, Bissler JJ. Hemolytic uremic syndrome in an adolescent with Fusobacterium necrophorium bacteraemia. Am J Kidney Dis 2001;37:e22

6 Brazier JS, Hall V, Yusuf E, Duerden BI. Fusobacterium necrophorium infections in England and Wales 1990-2000. J Med Microbiol 2002;51:269-72. 


\section{Pneumothorax following central venous catheterisation?}

\section{R J Parker, C G Wathen}

A chest radiograph performed to check the position of a central venous catheter in a patient appeared to show a pneumothorax. Intercostal drain insertion was prepared. Reassessment of the patient and a further radiograph confirmed that the "pneumothorax" was an artefact from a prominent skin fold due to the patient's body habitus.
A 73 year old woman with sepsis and renal impairment had a right subclavian central venous catheter placed as part of fluid resuscitation. The procedure was performed by an experienced operator. It was achieved "first pass" and thought to be uncomplicated. A supine chest radiograph performed to check position appeared to demonstrate a right sided pneumothorax (fig lA). An intercostal drain was considered. The patient's body mass index was $>30 \mathrm{~kg} / \mathrm{m}^{2}$, respiratory rate 18 breaths $/ \mathrm{min}$ and oxygen saturation $96 \%$ in room air. She was comfortable, speaking full sentences, and respiratory examination was unremarkable. The chest radiograph was re-evaluated. The pneumothorax is not so clear at the apex, and lung markings can be seen to the lung edge. A repeat erect chest radiograph (fig 1B) did not show the pneumothorax. We concluded that the abnormality is an artefact from a prominent skin fold due to the patient's habitus.

\section{Authors' affiliations}

R J Parker, C G Wathen, Department of Respiratory Medicine, Wycombe Hospital, High Wycombe, Buckinghamshire, UK

Competing interests: there are no competing interests

Correspondence to: Dr R J Parker, Thoracic Medicine Office, Wycombe Hospital, High Wycombe, Buckinghamshire HP1 1 2T, UK;

robertparker@doctors.org.uk

Accepted for publication 27 May 2004
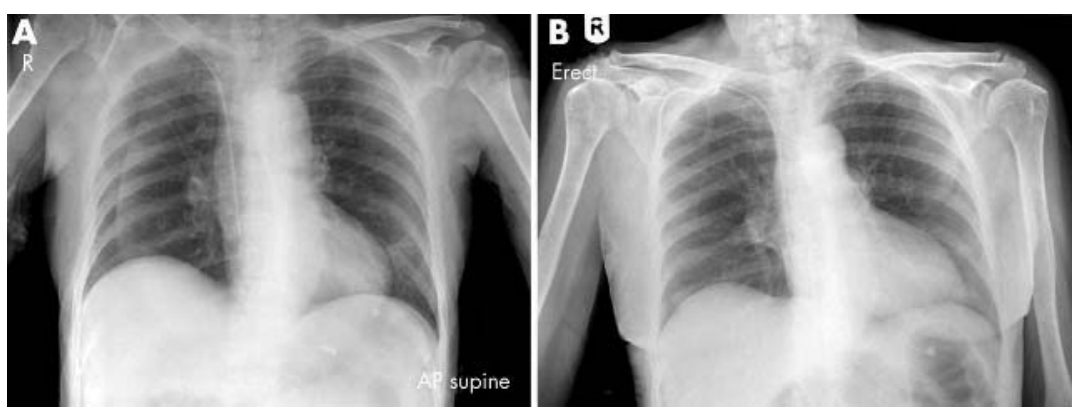

Figure 1 (A) Chest radiograph performed supine after central venous catheterisation; (B) erect radiograph showing the disappearance of the apparent pneumothorax. 


\section{Bilateral optic disc swelling; is a CT scan necessary?}

\section{U K Bhatt}

Emerg Med J 2005;22:827-830. doi: 10.1136/emj.2003.012971

\begin{abstract}
A 47 year old man sustained a head injury after tripping. He presented to the accident and emergency department next morning where head $x$ ray revealed no fractures. However, the casualty doctor found bilateral blurred optic disc margins on ophthalmoscopy. Although his head injury was classed as non-serious, an urgent computed tomography scan was ordered and an ophthalmic opinion sought. After detailed retinal examination, the ophthalmologist made a diagnosis of bilateral optic nerve head drusen (ONHD), which was confirmed by a B-scan ultrasound. The patient was advised not to drive (due to constricted visual fields associated with ONHD) and to inform his siblings of his condition so they could also be assessed. In cases of apparent optic disc swellings, it is essential to distinguish between true and pseudo-papillo-oedema to avoid subjecting patients to unnecessary neuroimaging procedures and associated exposure to radiation.
\end{abstract}

A 47 year old man sustained a head injury after tripping over and hitting his head against a pillar. He developed a small swelling at the site of injury on his forehead just above the lateral end of the right eyebrow. He was seen in the accident and emergency (A\&E) department the next morning because of pain and swelling at the site of injury. He did not have any sign of intracranial injury and the head radiographs did not show any fractures, thus the injury was classified as non-serious. However, during examination the A\&E doctor noted bilaterally blurred optic discs on ophthalmoscopy. A case of papillo-oedema secondary to raised intracranial pressure (ICP) was suspected, and an urgent brain computed tomography (CT) scan was advised to rule out any intracranial pathology. An urgent ophthalmic consultation was also requested.

Examination by the ophthalmologist revealed corrected visual acuity in both eyes to be 6/6 (using Snellen's visual acuity chart). Both pupils were equal and reacting to light, and no relative afferent pupillary defect was noted. Anterior segment examination showed slight conjunctival injection in both eyes. In both eyes, the corneas, anterior chambers, and lenses were normal. Fundoscopic examination showed discs with clear but slightly crenated margins and obliteration of the physiological optic disc cupping. Venous pulsations were present in both eyes. Retinal vasculature showed premature branching on emerging from the discs (fig 1A, B). Visual field examination by confrontation showed grossly restricted peripheral visual fields in both eyes. A diagnosis of bilateral optic nerve head drusen (ONHD) was made, which was subsequently confirmed by autofluorescence (fig 2A, B) and a B scan ultrasound (fig 3), in the ophthalmic outpatient department. Addtionally, a formal visual field examination using a Humphries visual field analyser was performed, which confirmed severe restriction of visual fields in both eyes (fig 4A, B). In the absence of any other signs of raised ICP, the CT brain scan was cancelled. The patient was advised subsequently not to drive, and the Driver and Vehicle Licensing Agency was informed. He was also advised to inform his siblings of his condition so that they could have an ophthalmic examination to exclude optic disc drusens.

\section{DISCUSSION}

ONHDs are yellow opalescent hyaline excrescences derived from calcified axonal debris present on the surface of the disc or buried in it. They are present in $0.3 \%$ of the population and are bilateral in about $75 \%$ of cases. They can be sporadic, congenital, or inherited as an autosomal dominant trait with incomplete penetrance. In early age, they remain deeply embedded deep in the nerve (but anterior to the lamina cribrosa) and not visible by ophthalmoscopy. With increasing age, they become more visible and recognisable as they come onto the surface, by the third or fourth decade of life. The optic nerve head in these cases is typically small and full, mimicking papillo-oedema. Drusens are recognised on the disc as a small, mulberry-like mass or a waxy elevation composed of a conglomerate of smaller masses. In doubtful cases if diagnosis needs to be confirmed, a simple ultrasound, autofluorescence using red free light (when viewing through a fundus camera), or a fundus fluorescin angiography (FFA) is diagnostic. In cases of children with anomalously elevated discs without visible drusen, examination of family members
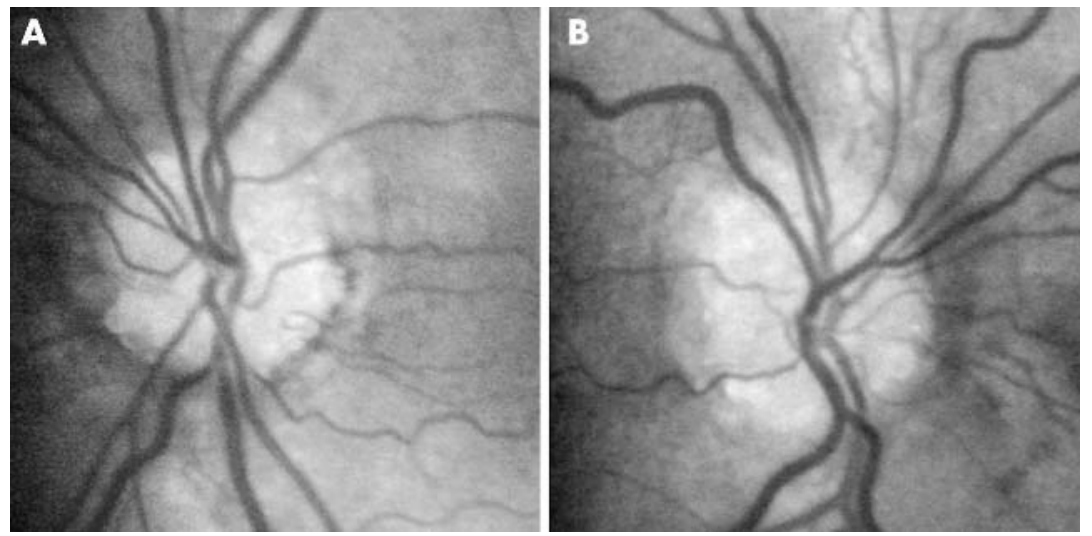

Figure 1 Drusen disc: $(\mathrm{A})$ right eye; (B) left eye. 

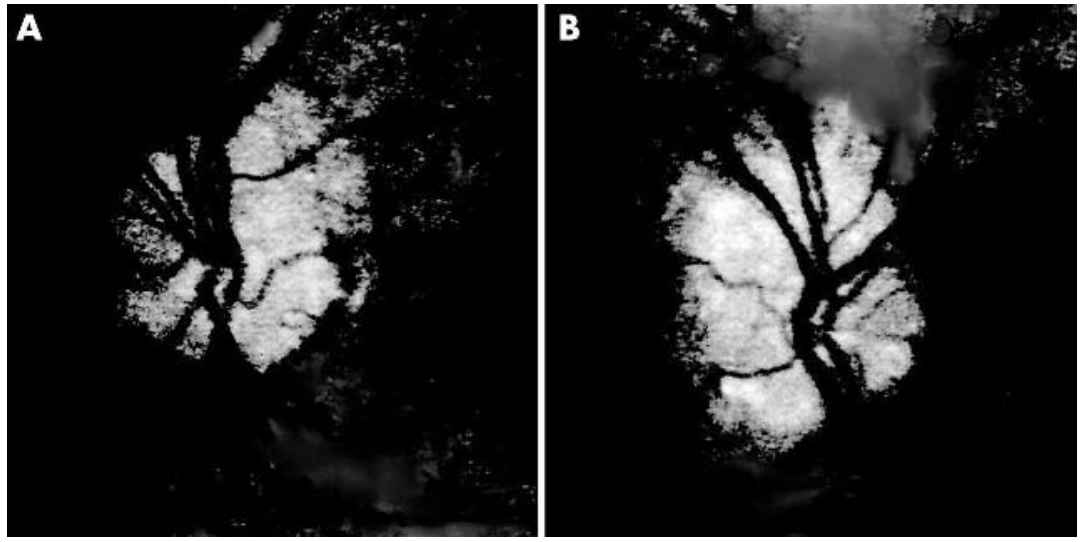

Figure 2 Autofluorescence: (A) right eye; (B) left eye.

is essential if the distinction between true papillo-oedema and pseudo-papillo-oedema in the child is in doubt. ${ }^{1-3}$

Three patterns of visual defect may be found: (a) enlargement of the blind spot (60\% of cases); $(b)$ arcuate or nerve fibre bundle defects producing sector cuts, ring scotomas and paracentral scotomas; or (c) generalised irregular field constrictions (as in this patient). ${ }^{2}$ Visual fields are an important method of documenting and monitoring optic nerve compromise secondary to ONHD, but they are neither uniform nor diagnostic.

Ultrasonography is probably the best ancillary test to perform in adults with ONHD, demonstrating calcification within the disc. It should be performed on all adult patients presenting with elevated optic discs that are not definitively identifiable. However, it must be kept in mind that optic disc drusen are generally not calcified in children and adolescents. Hence, ultrasonography is not very helpful in diagnosing these cases.

Retinal vasculature in patients with optic disc drusen may show increased tortuosity and premature branching. Optic disc drusens may also cause spontaneous haemorrhage, which may appear peripapillary flame shaped, either intravitreal or subretinal. Incidence of retinal haemorrhage in optic disc drusen is $2-10 \%$. It may also give rise to complications such as ischaemic optic neuropathy or choroidal neovascularisation, which cause significant visual loss. ${ }^{2-4}$

The majority of cases of disc drusens are harmless and remain asymptomatic. As a rule, central vision is intact.

Autofluorescence is an important property of ONHD. It is best demonstrated by visualising through fluorescein

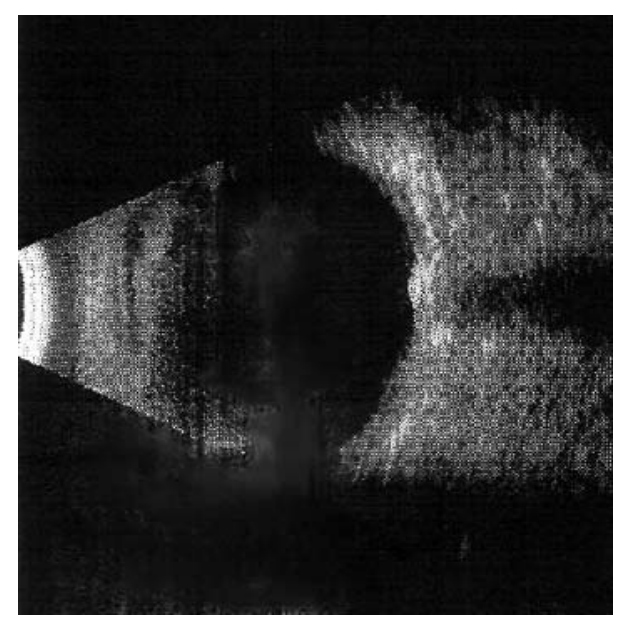

Figure 3 Ultrasound showing increased signal at the drusen disc. angiography filters (prior to the injection of fluorescein). Using FFA, the drusens become stained during the late stages of the angiogram. In ONHD, disc is typically "normal" pink to pinkish yellow in colour, rather than waxy pale or hyperaemic. In addition, a spontaneous venous pulsation is present in about $80 \%$ of patients with ONHD, but is absent in cases of true disc oedema. Most importantly, while the disc margins may be irregular in ONHD, they are rarely blurred or obscured.

The development of papillo-oedema after head injury is caused by elevated ICP, but it is very important to realise that it takes time ( 3 days to 3 weeks) for papillo-oedema to develop even after raised ICP is documented. In one study, papillo-oedema was seen in only $3.5 \%$ of 426 patients with severe trauma in whom the frequency of elevated ICP exceeded 50\%. ${ }^{5}$ Therefore, although papillo-oedema is a positive sign of elevated ICP, more importance should be given to other specific signs of elevated ICP.

\section{Competing interests: none declared}

The author was involved in examining the patient as an ophthalmologist

Correspondence to: Dr U K Bhatt, Department of Ophthalmology, Leicester Royal Infirmary, Infirmary Square, Leicester LE1 5WW, UK; bhattuday@yahoo.com

Accepted for publication 3 May 2004

\section{Key learning points}

- This case highlights the importance of this relatively benign cause of pseudo-papillo-oedema.

- Papillo-oedema secondary to head injury develops very slowly over days to weeks, and it must be remembered that it is usually accompanied by other signs or symptoms of raised ICP.

- If disc swelling is seen very soon after the head injury, some other cause must be suspected and should be looked for.

- Presence of swollen optic discs does not necessarily mean raised ICP and therefore other causes should always be considered before coming to a final conclusion based on all relevant clinical findings.

- By being aware of the existence of this cause of pseudo-papillo-oedema and referring these patients to ophthalmologists, physicians can avoid subjecting patients to unnecessary neuroimaging procedures and associated exposure to radiation. 
A
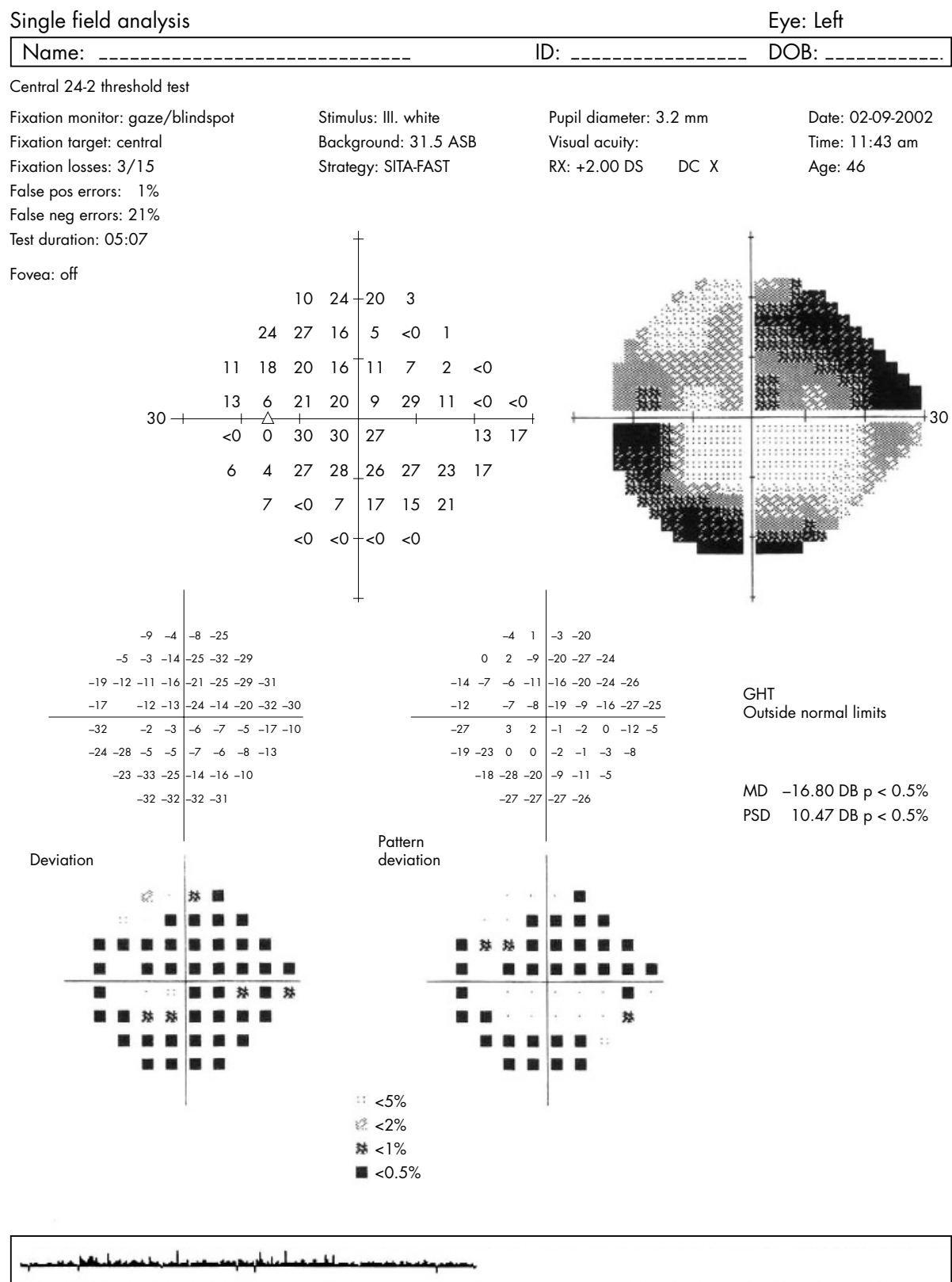

Figure 4A Visual field, showing generalised constriction, right eye.

\section{REFERENCES}

1 Kanski JJ. Optic disc drusen. In: Clinical ophthalmology, 5th ed. London: Butterworth Heinemann, 2003:613-16.

2 Pavan-Langstan D. Pseudopapilledema. In: Manual of ocular diagnosis and therapy, 4th ed. Boston: Little, Brown \& Co, 1996:350.
3 Auw-Haedrich C, Staubach F, Witschel H. Optic disk drusen. Surv Ophthalmol 2002;47:515-32.

4 Mustonen E. Pseudopapilloedema with and without verified optic disc drusen. A clinical analysis I. Acta Ophthalmol (Copenh) 1983;61:1037-56.

5 Selhorst JB, Gudeman SK, Butterworth JF IV, et al. Papilledema after acute head injury. Neurosurgery 1985;16:357-63. 
B

\begin{tabular}{l} 
Single field analysis \\
\hline Name:
\end{tabular}

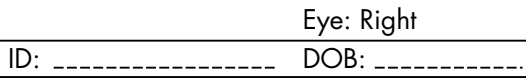

Central 24-2 threshold test

Fixation monitor: gaze/blindspot

Fixation target: central

Fixation losses: $4 / 14$

False pos errors: $0 \%$

False neg errors: $0 \%$

Test duration: 05:56

Fovea: off

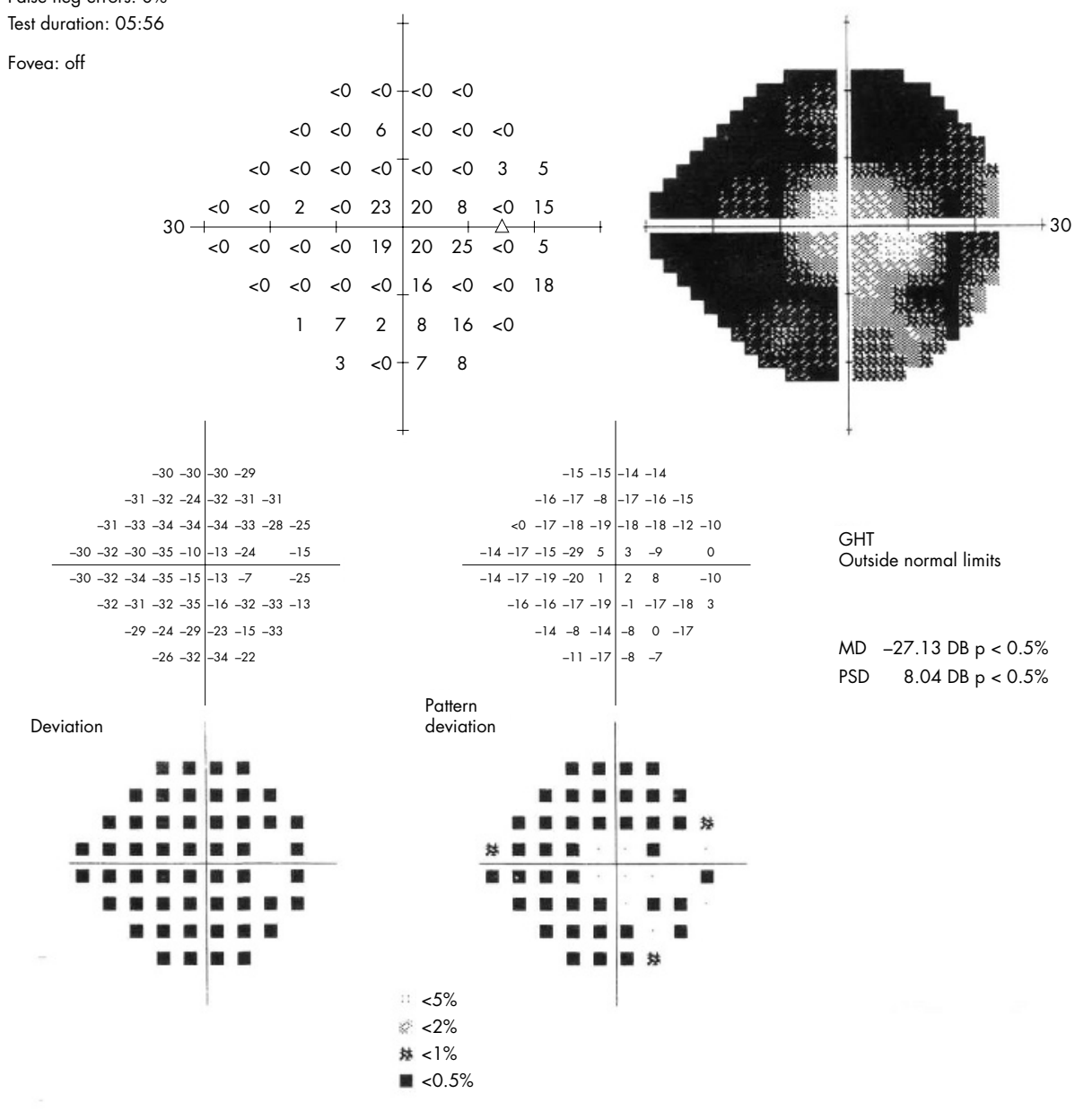

$\begin{array}{lll}\text { Stimulus: III. white } & \text { Pupil diameter: } 3.1 \mathrm{~mm} & \text { Date: 02-09-2002 } \\ \text { Background: } 31.5 \text { ASB } & \text { Visual acuity: } & \text { Time: } 11: 34 \mathrm{am} \\ \text { Strategy: SITA-FAST } & \text { RX: +2.00 DS DC X } & \text { Age: } 46\end{array}$

Foved: off

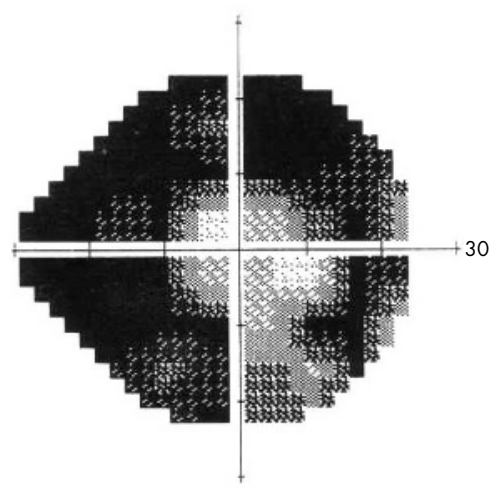

$-31-32-24-32-31-31$

$-33-34-34-34-33-28-25$

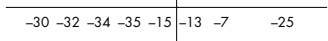

$-31-32-35-16-32-33-13$

$\begin{array}{lllll}-24 & -29 & -23 & -15 & -33\end{array}$

(n)

iation

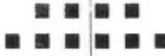

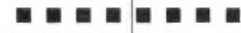

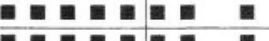

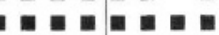

a

-

Pupil diameter: $3.1 \mathrm{~mm} \quad$ Date: 02-09-2002

X: +2.00 DS DC X Age: 46

$M D-27.13 D B p<0.5 \%$

PSD $\quad$ 8.04 DB $p<0.5 \%$

\section{Pattern}

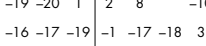

\begin{tabular}{ll|lll}
-8 & -14 & -8 & 0 & -17
\end{tabular}

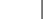

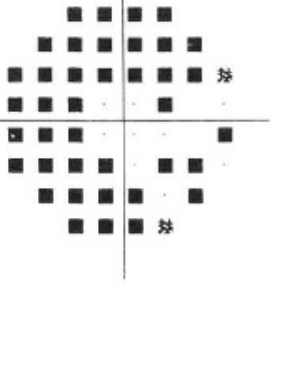




\title{
Purtscher's retinopathy after fracture dislocation of shoulder joint
}

\author{
H Nayak, S Harun, P Palimar
}

Emerg Med J 2005;22:831-832. doi: 10.1136/emj.2004.015503

Purtscher described sudden blindness in patients with severe head trauma due to a remote retinopathy, characterised by bilateral retinal haemorrhages, cotton wool spots, and optic disc swelling seen on fundoscopy. A similar retinopathy has been reported in compressive chest trauma, long bone fractures, and acute pancreatitis. It is less well recognised that Purstcher's retinopathy can occur unilaterally and following less severe trauma. We present a case of unilateral remote traumatic retinal angiopathy following a fracture dislocation of the shoulder joint.

$\mathrm{P}$ urtscher described sudden blindness in patients with severe head trauma due to a remote retinopathy, characterised by bilateral retinal haemorrhages, cotton wool spots, and optic disc swelling seen on fundoscopy. ${ }^{1} \mathrm{~A}$ similar retinopathy has been reported in compressive chest trauma, long bone fractures, and acute pancreatitis. ${ }^{2}$ The onset of symptoms is typically within 2 days, with patients complaining of decreased vision, often from 6/60 to only being able to count fingers. The retinal lesions gradually settle down over weeks and then the retina may appear normal. In severe cases, pigmentary changes and optic atrophy may ensue. Arteriolar occlusion due to embolisation is considered as the main mechanism involved in the pathogenesis of this condition. Air, fat, granulocyte, or other blood product aggregates formed after complement activation have all been suggested as the emboli responsible for arteriolar occlusion..$^{3-5}$ It is less well recognised that Purstcher's retinopathy can occur unilaterally ${ }^{3}$ and following less severe trauma. ${ }^{6}$ We present a case of unilateral remote traumatic retinal angiopathy following a fracture dislocation of the shoulder joint.

\section{CASE REPORT}

A 50 year old man presented to the eye clinic with blurred vision in his right eye 2 days after a $50 \mathrm{mph}$ motorcycle accident. He had sustained a dislocation of his right shoulder joint, along with an avulsion fracture of the greater tuberosity of the humerus. The dislocation had been relocated on the same day. He had not suffered ocular or periorbital trauma. On examination, he had a vision of 6/12 in the right eye and $6 / 6$ in the left. Fundoscopy showed scattered cotton wool spots in the right eye, with a few retinal haemorrhages in the macular area (fig 1). The left eye was normal. Visual field testing revealed a right sided central scotoma (fig 2). Fundus fluorescein angiography demonstrated decreased capillary perfusion of the right macular area and no treatment could be offered. Two months later his vision had improved to 6/9, the cotton wool spots and haemorrhages had resolved, but

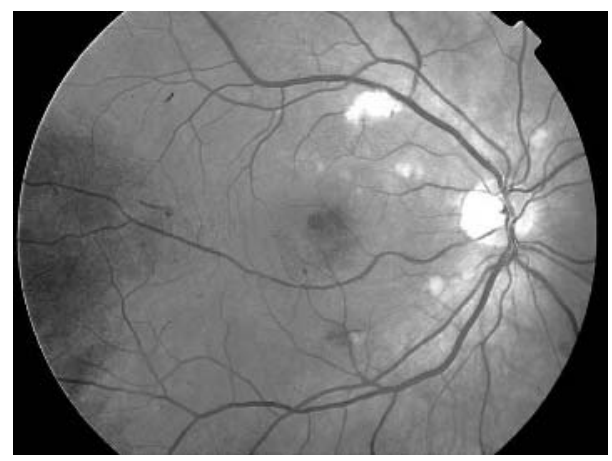

Figure 1 Retinal photograph, right eye.

the central scotoma was still present.

\section{CONCLUSION}

Patients who have had traumatic injuries and develop visual symptoms need an ophthalmological referral for dilated retinal examination in order to identify signs of Purstcher's retinopathy. Although treatment may not be successful, these findings may have medicolegal implications. The patient needs to be counselled as to a guarded visual prognosis.

\section{Authors' affiliations}

H Nayak, S Harun, P Palimar, Department of Ophthalmology, Kendrick Wing, Warrington Hospital, Lovely Lane, Warrington, UK

Correspondence to: Dr H Nayak, Department of Ophthalmology, University Hospital Wales, Warrington Hospital, Heath, Cardiff CF14 4XN, UK; nayaks@tesco.net

Accepted for publication 22 June 2004

\section{REFERENCES}

1 Purtsher O. Angiopathia retinae traumatica: Lymphorrhagien des Augengrundes. Graefes Arch Klin Exp Ophthalmol 1912;82:347-71.

2 Gass JDM. Stereoscopic atlas of macular diseases: diagnosis and treatment, 2nd ed. St. Louis: Mosby, 1987.

3 Burton TC. Unilateral Purtscher's retinopathy. Ophthalmology 1980;87:1096-105

4 Roden D, Fitzpatrick G, O'Donoghue $H$, et al. Purtscher's retinopathy and fat embolism. Br J Ophthalmol 1989;73:677-9.

5 Behrens-Baumann W, Scheurer G, Schroer H. Pathogenesis of Purtscher's retinopathy. An experimental study. Graefes Arch Clin Exp Ophthalmol 1992;230:286-91.

6 Peled M, Rubin A, Pikkel J, et al. Purtscher's retinopathy associated with mandibular fracture: a case report. J Trauma 1996;40:141-3. 


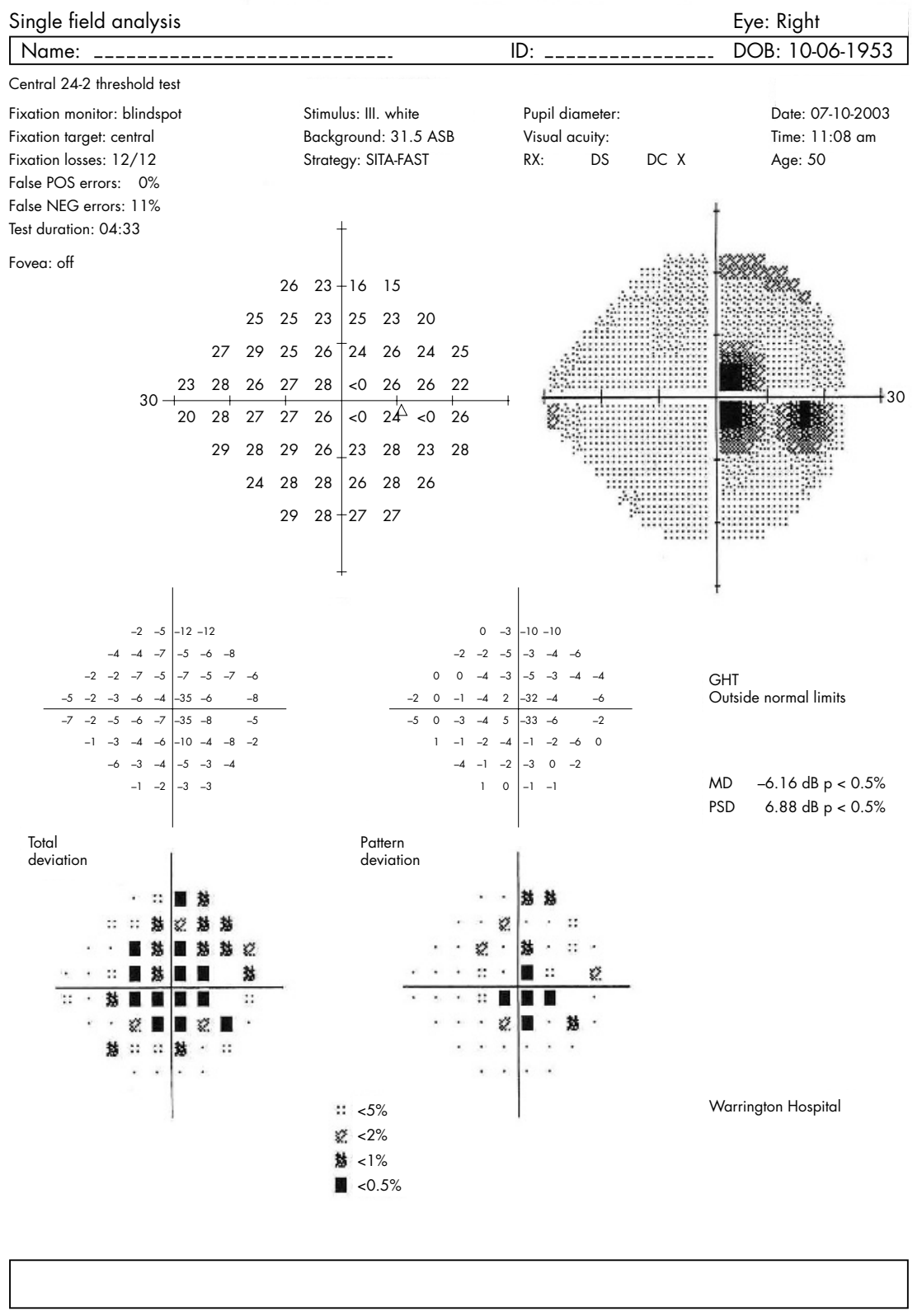

Figure 2 Computerised visual field, right eye.

\section{Modified release verapamil induced cardiogenic shock}

\section{U Nanda, A Ashish, H J F Why}

Cardiogenic shock due to acute myocardial infarction is commonly seen in the accident and emergency department. Refractory cardiogenic shock has been reported after a therapeutic dose of modified release verapamil with concomitant use of beta blocker, metoprolol, but not after a single therapeutic oral dose of modified release verapamil alone. We report what we believe to be the first case of potentially life threatening cardiogenic shock resulting from the myocardial depressant effect of a single therapeutic oral dose of modified release verapamil. The patient made a dramatic recovery minutes after an injection of intravenous calcium chloride. The case is a reminder of the negative inotropic effect of verapamil and how it should be treated. 
A 58 year old, previously fit man presented with a 6 hour history of vomiting and lightheadedness. There was no preceding history of chest pain or breathlessness. The only medical history of note was chronic atrial fibrillation treated with digoxin and warfarin. Twenty hours prior to admission, he had sought a second cardiological opinion for his irregular heartbeat, and had been prescribed modified release verapamil $240 \mathrm{mg}$ once daily, in addition to his previous medications for better ventricular rate control. He had taken the first dose 12 hours prior to presentation.

On admission, he was alert but his peripheries were cold and cyanosed. Radial pulse was feeble but regular, at a rate of $52 / \mathrm{min}$. He was hypotensive at $85 / 40 \mathrm{mmHg}$, and jugular venous pressure was not raised. Systemic examination was otherwise unremarkable with specifically no evidence of heart failure, pericardial tamponade or obvious blood loss. ECG showed atrial fibrillation with ventricular rate of $44 / \mathrm{min}$ and chest $x$ ray revealed moderate cardiomegaly with clear lung fields. Full blood count, electrolytes, and liver function tests were normal, but urea and creatinine were raised, at $13 \mathrm{mmol} / \mathrm{l}$ and $248 \mathrm{umol} / \mathrm{l}$ respectively (both had been normal 2 days earlier). Serum digoxin concentration was subtherapeutic at $0.6 \mu \mathrm{g} / \mathrm{l}$. Both creatine phosphokinase and troponin I concentrations were normal.

The patient was initially managed with intravenous atropine $600 \mu \mathrm{g}$ and intravenous colloids, without any change to his existing rhythm and blood pressure. Intermittent long pauses were then noticed on his rhythm strip, with a further drop in blood pressure. Temporary pacing was considered and urgent cardiological opinion was sought. Transthoracic echocardiography was performed immediately, which demonstrated globally poor left ventricle function without any evidence of pericardial tamponade. Acute aortic dissection was unlikely, based on a normal aortic root and abdominal aorta. Twelve months previously, his echocardiogram had shown good left ventricular function with normal cardiac dimensions.

Over the next 15 minutes, the patient deteriorated rapidly, becoming confused and less responsive with unrecordable $\mathrm{SaO}_{2}$ despite high flow oxygen. His blood pressure was also unrecordable and all major pulses became impalpable, even though the cardiac monitor showed a ventricular rate of $94 / \mathrm{min}$. He was transferred immediately to the intensive therapy unit (ITU) for close monitoring, respiratory and inotropic support. Just before transfer to ITU, $10 \mathrm{ml}$ of $10 \%$ calcium chloride was administered intravenously in view of the possibility of calcium channel blocker toxicity. Within a few minutes, he made a dramatic recovery. By the time of his arrival to ITU, he had a good volume radial pulse and a blood pressure of 110/ $70 \mathrm{mmHg}$, and was more alert and responsive. Cardiac index measurement was normal, and a repeat echocardiography showed much improved left ventricular contractility. The remainder of his hospital stay was uneventful. His renal function returned to normal and he was discharged 2 days later on digoxin and warfarin.

\section{DISCUSSION}

Verapamil is a non-dihydropyridine calcium channel blocker chemically related to the opium alkaloid, papaverine. ${ }^{1}$ It is used to treat hypertension, angina, and supraventricular dysrhythmias. It inhibits voltage dependent L-gated calcium channels, which leads to vascular smooth muscle relaxation with negative inotropic and chronotropic effects on the

Abbreviations: ITU, intensive therapy unit; JVP, jugular venous pressure

heart. $^{2}$ It also acts indirectly to cause coronary artery dilatation and reduced myocardial oxygen demand. In response to vasodilatation, there is a baroreceptor mediated reflex increase in beta adrenergic tone.

This case illustrates undue sensitivity to the profound myocardial depressant effect of a therapeutic oral dose of modified release verapamil. The clinical features were typical of a non-dihydropyridine calcium channel blocker. Verapamil induced myocardial depression was not considered in the first instance as it was thought unlikely to have resulted from a single therapeutic dose of an oral modified release preparation.

Verapamil is known to increase the serum digoxin concentration and to potentiate digoxin induced atrioventricular block. However, digoxin concentration at presentation in our patient was subtherapeutic, and echocardiography during the profound hypotensive episode showed globally poor left ventricle function, which improved dramatically following intravenous injection of calcium chloride. These features could not be explained by an enhanced effect of digoxin, following interaction with verapamil.

Published reports describe cardiac arrest after the use of a single dose of intravenous verapamil when used to treat supraventricular dysrhythmias ${ }^{3}$ and a delayed severe hypotensive episode 12 hours after ingestion of an overdose of modified release verapamil. ${ }^{4}$ Refractory cardiogenic shock and complete heart block has been reported 2 days after a therapeutic dose of modified release verapamil with concomitant use of metoprolol. The patient remained hypotensive with complete heart block, even after multiple doses of intravenous atropine as well as high doses of inotropes such as dopamine and dobutamine. However, shortly after a dose of intravenous calcium chloride, the refractory hypotension and complete heart block resolved. ${ }^{5}$

\section{CONCLUSION}

The myocardial depressant effect of non-dihydropyridine calcium channel blockers (verapamil and diltiazem) should always be considered in patients taking these drugs who display high grade atrioventricular block, severe left ventricle dysfunction, or cardiogenic shock irrespective of dose or duration of treatment. Early treatment with $10 \mathrm{ml}$ of $10 \%$ calcium chloride given intravenously is the key to management of such cases and can prove life saving.

\section{Authors' affiliations \\ U Nanda, University Hospital of North Staffordshire, Stoke on Trent, UK A Ashish, H J F Why, Queens Hospital, Burton on Trent, UK \\ Competing interests: none declared}

Correspondence to: $\operatorname{Dr} U$ Nanda, Department of Respiratory Medicine, Royal Shrewsbury Hospital, Shrewsbury SY3 8XQ, UK; uttam_nanda@ yahoo.com

Accepted for publication 19 June 2004

\section{REFERENCES}

1 In: Hamer John, ed. Drugs for heart disease, 2nd ed; chapter 33.5.

2 Scholz H. Pharmacological aspects of calcium channel blockers. Cardiovasc Drugs Ther 1997;10(suppl 3):869-72.

3 Lawrenson JB, Okreglicki AM, Millar RN. Cardiovascular collapse due to intravenous verapamil in two patients with persistant atrial tachycardia. S Afr Med J 1995:85(suppl):1236-8.

4 Asraf M, Chaudhary K, Nelson J, et al. Massive overdose of sustained release verapamil:a case report and review of literature. Am J Med 1995;310:258-63.

5 Lee DW, Cohan B. Refractory cardiogenic shock and complete heart block after verapamil SR and metoprolol. Angiology 1995;46:517-19. 


\title{
Eosinophilic gastroenteritis presenting as relapsing severe abdominal pain and enteropathy with protein loss
}

\author{
H-H Lin, C-H Wu, L-S Wu, R-Y Shyu
}

Emerg Med J 2005;22:834-835. doi: 10.1136/emj.2004.016337

Eosinophilic gastroenteritis (EG) is an uncommon gastrointestinal tract disease, and diagnosis can be difficult. A combination of acute or recurrent abdominal pain with peripheral hypereosinophilia suggests the diagnosis. Surgery in patients with these features might therefore be avoided. Physicians must maintain a high index of suspicion and a working knowledge of the natural history of EG in order to establish the proper diagnosis. We present the case of a young man with EG who presented with relapsing severe abdominal pain and enteropathy with protein loss.

E osinophilic gastroenteritis (EG) is an uncommon gastrointestinal tract disease. The need for histological confirmation makes diagnosis problematic. The symptoms and signs of the condition can simulate an acute abdomen. Reports describe the disease mimicking acute appendicitis, ${ }^{1}$ an obstructing caecal mass, ${ }^{2}$ pancreatitis, ${ }^{3}$ cholecystitis, ${ }^{4}$ giant refractory duodenal ulcer, ${ }^{5}$ and intussusception. $^{6}$ A combination of acute or recurrent abdominal pain with peripheral hypereosinophilia suggests the diagnosis. Surgery in patients with these features might therefore be avoided. Physicians must maintain a high index of suspicion and a working knowledge of the natural history of EG in order to establish the proper diagnosis. We present the case of a young man with EG who presented with relapsing severe abdominal pain and enteropathy with protein loss.

\section{CASE PRESENTATION}

A 22 year old man presented to our emergency department with severe abdominal pain. Seven years before, he began developing abdominal pain, bloating, vomiting, and watery diarrhoea that lasted 3-4 months each winter. His symptoms remitted without treatment so he did not seek medical care. More recently, the symptoms had worsened and he had lost $13 \mathrm{~kg}$ over the previous 3 years. He had no personal or family history of allergic disorders, asthma, hay fever, drug hypersensitivity, or eczema. Past medical and surgical histories and personal habits were non-contributory.

Upon admission, vital signs were normal. A physical examination was unremarkable except for a hyperactive bowel sound and diffuse abdominal tenderness. Laboratory valuses were: haemoglobin, $124 \mathrm{~g} / \mathrm{l}$ (normal range 135$180 \mathrm{~g} / \mathrm{l}$ ); mean corpuscular volume $63.5 \times 10^{-15} \mathrm{l}$ (normal range $79-100 \times 10^{-15} 1$ ); leukocyte count, $7.94 \times 10^{9} / 1$ (normal range $4.5-11 \times 10^{9} / 1$ ); platelet count, $364 \times 10^{9} / 1$ (normal range $\left.150-400 \times 10^{9} / 1\right)$, with a differential count of $52 \%$ neutrophils (normal range, 40-74\%), 25\% lymphocytes (normal range $19-48 \%$ ) and $13 \%$ eosinophils (normal range 0-7\%). Stool samples were examined twice and no ova or parasites were found. No faecal leucocytes were detected and stool culture was negative. Serum electrolytes, blood urea nitrogen, creatinine, and liver function tests were within normal limits. The serum albumin level (18 g/l) was low (normal range $39-51 \mathrm{~g} / \mathrm{l})$. Proteinuria was absent.

The patient's abdominal pain and recent weight loss prompted his admission for serial abdominal examinations and urgent investigation. An abdominal sonogram was normal. Barium visualisation of the upper gastrointestinal tract showed diffuse mucosal fold thickening involving the duodenum, jejunum, and ileum. An upper gastrointestinal endoscopy with gastric mucosal biopsy was performed. Histological sections of the gastric tissue demonstrated foci of active gastritis with eosinophilic infiltration (fig 1). We checked his gut $\alpha \mathrm{l}$-antitrypsin clearance ( 24 hour volume of faeces $\times$ stool concentration of $\alpha \mathrm{l}$-antitrypsin $\div$ serum $\alpha \mathrm{l}$ antitrypsin concentration). The clearance value of $15 \mathrm{~mL}$ in $24 \mathrm{~h}$ was above the normal range $(<13 \mathrm{ml} / 24 \mathrm{~h})$.

A diagnosis of enteropathy with protein loss was made on the basis of the increased $\alpha \mathrm{l}$-antitrypsin clearance. Mucosal EG with protein losing enteropathy was confirmed. The patient was treated with prednisone and placed on an elimination diet, and his abdominal pain and diarrhoea improved over the next 2 weeks.

\section{DISCUSSION}

EG is a rare inflammatory disease characterised by abdominal pain with bloating, nausea and diarrhoea, eosinophilic infiltration of the gastrointestinal tract, and the absence of extraintestinal involvement and parasitic disease. ${ }^{7}$ Peripheral blood hypereosinophilia is evident in $20-90 \%$ of patients with the disease. ${ }^{8}$ The aetiology of EG is unknown, but speculation has focused on the selective release of eosinophil major proteins as a prelude to intestinal epithelial damage. The disease can affect any age group. ${ }^{10}$

Clinical features of EG correlate with the depth of eosinophilic infiltration in the bowel wall and the location of lesions within the gut. ${ }^{11} \mathrm{EG}$ is therefore classified into mucosal, muscular, and serosal subtypes. ${ }^{11}$ The mucosal form, which is most common, ${ }^{11}$ presents like most other inflammatory gastrointestinal disorders, with abdominal pain, diarrhoea, anorexia, bloating, nausea, and vomiting. Patients may have protein losing enteropathy. There is a frequent association of the disease with seasonal allergy, food sensitivity, eczema, allergic rhinitis, atopy, and bronchial asthma. ${ }^{12}$ The muscular subtype typically presents with pyloric or intestinal obstruction. ${ }^{13}$ The eosinophilic infiltrates often involve the muscularis layer of the stomach but can also involve the small intestine.$^{10}$ Serosal EG is the least common subtype ( $10 \%$ of cases). It produces eosinophilic peritonitis and ascites. ${ }^{14}$

Peripheral eosinophilia is found in a variable number of EG patients. The magnitude of the eosinophilia seems to correlate with the severity of symptoms. Iron deficiency anaemia may be evident. Elevated IgE, allergy evaluation by skin and serum radioallergosorbent tests, hypoalbuminaemia, increased faecal $\alpha-1$ anti-trypsin levels, elevated erythrocyte sedimentation rate and the presence of eosinophils in ascites fluid provide further diagnostic support. ${ }^{11}$ Mild to moderate steatorrhoea is present in approximately $30 \%$ of patients. A sterile exudative peritoneal effusion with up to $95 \%$ eosinophils is characteristic of serosal eosinophilic gastroenteritis. ${ }^{15}$

Abbreviation: EG, eosinophilic gastroenteritis 
Radiological tests are typically not helpful in diagnosing EG. Barium studies may show a variable degree of antral stenosis with mucosal irregularity, gastric pseudopolyposis, or thickened mucosal folds due to oedema. ${ }^{7}$ The most common computed tomography finding is nodular and irregular fold thickening in the distal stomach and proximal small bowel. Ascites fluid is usually detected in patients with serosal layer involvement. The gastric antrum and contiguous small bowel are affected in about $70 \%$ of patients. ${ }^{8}$ Uncommonly, only the small bowel is affected and, rarely, only the colon is affected. Mucosal biopsies are not diagnostic in $10 \%$ of cases, ${ }^{16}$ owing to inappropriate sampling or a lack of mucosal involvement.

EG is characterised by a patchy distribution of lesions, necessitating the need for at least six biopsy specimens. Surgical biopsy is needed in patients with muscular or serosal EG subtypes. ${ }^{11}$ Eosinophils are a normal component of the gastrointestinal mucosa, therefore greater than 19 eosinophils per high power field are necessary for diagnosis. ${ }^{7}$ Large numbers of eosinophils are often present in the muscularis and serosal layers. The localised eosinophilic infiltrates may cause crypt hyperplasia, epithelial cell necrosis and villous atrophy. ${ }^{7}$

\section{Treatment}

Once the diagnosis of EG is confirmed, it is useful to test for food allergy, as an elimination diet may provide symptomatic relief. ${ }^{10}$ Corticosteroids are the mainstay of therapy. Symptom remission usual occurs within a few weeks of treatment initiation. ${ }^{10}$ The standard treatment regimen is $20-40 \mathrm{mg}$ (paediatric dose $0.14-2 \mathrm{mg} / \mathrm{kg}$ ) of prednisone daily in divided doses for 7-10 days followed by a taper over 2-3 months. ${ }^{8}{ }^{14}$ Patients with refractory or relapsing symptoms are usually maintained on long term, low dose corticosteroids.

Three alternative treatments can be used. ${ }^{8}$ Sodium cromoglycate, a mast cell stabiliser effective in the treatment of allergies or bronchial asthma, gives variable results, and the recommended dosage for oral administration varies between 100 and $300 \mathrm{mg}$ per dose four times daily. ${ }^{10}$ Ketotiphene, an antihistaminic agent and mast cell membrane stabiliser, produces clinical improvement, normal eosinophil values and disappearance of the intestinal eosinophilic infiltrate within 1-4 months when administered at $2-4 \mathrm{mg}$ daily. ${ }^{17}$ Montelukast, a selective and competitive leukotriene receptor

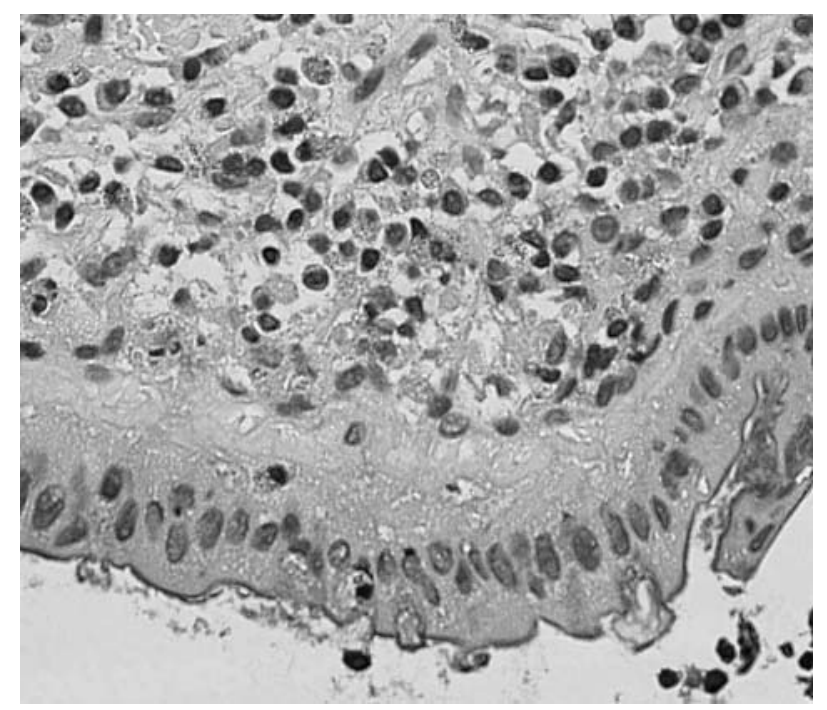

Figure 1 Biopsy showing the histological sign of eosinophilic infiltration in the lamina propria (haematoxylin and eosin staining; original magnification $\times 100$ ). antagonist, can be a successful corticosteroid sparing agent. ${ }^{18}$ The treatment regimen is $10 \mathrm{mg}$ (paediatric dose $5 \mathrm{mg}$ ) of montelukast daily.

\section{CONCLUSION}

The diagnosis of EG is challenging because it is a rare disease, is not often considered, presents with non-specific signs and symptoms, is marked by periods of remission and relapse, and the final diagnosis requires histological confirmation. ${ }^{7}$ However, EG should be considered in the differential diagnosis of patients with unexplained acute or recurrent abdominal pain and peripheral hypereosinophilia. ${ }^{11}{ }^{19}$ Stool examinations to exclude parasitic infection are mandatory. Endoscopy with multiple biopsies is required to confirm the diagnosis.

\section{Authors' affiliations}

H-H Lin, C-H Wu, R-Y Shyu, Department of Internal Medicine, TriService General Hospital, National Defense Medical Center, Taipei, Taiwan

L-S Wu, Department of Internal Medicine, Army Forces Hualien General Hospital, Hualien, Taiwan

Competing interests: none declared

Correspondence to: Dr R-Y Shyu, Division of Gastroenterology,

Department of Medicine, Tri-Service General Hospital, No. 325, Sec 2

Cheng-Kung Rd., Neihu 114, Taipei, Taiwan, China; redstone120@ yahoo.com.tw

Accepted for publication 8 July 2004

\section{REFERENCES}

1 Tran D, Salloum L, Tshibaka C, et al. Eosinophilic gastroenteritis mimicking acute appendicitis. Am Surg 2000;66:990-2.

2 Redondo Cerezo E, Moreno Platero JJ, Garcia Dominguez E, et al. Comments to a report: eosinophilic gastroenteritis presenting as an obstructing cecal mass: review literature and our own experience. Am J Gastroenterol 2000;95:3655-7

3 Maeshima A, Murakami H, Sadakata $\mathrm{H}$, et al. Eosinophilic gastroenteritis presenting with acute pancreatitis. J Med 1997;28:265-72.

4 Jimenez-Saenz M, Villar-Rodriguez JL, Torres $Y$, et al. Biliary tract disease: a rare manifestation of eosinophilic gastroenteritis. Dig Dis $\mathrm{Sci}$ 2003;48:624-7

5 Markowitz JE, Russo P, Liacouras CA. Solitary duodenal ulcer: a new presentation of eosinophilic gastroenteritis. Gastrointest Endosc 2000;52:673-6

6 Huang FC, Ko SF, Huang SC, et al. Eosinophilic gastroenteritis with perforation mimicking intussusception. J Pediatr Gastroenterol Nutr 2001;33:613-15.

7 Blackshaw AJ, Levison DA. Eosinophilic infiltrates of the gastrointestinal tract. J Clin Pathol 1986;39:1-7

8 Talley NJ, Shorter RG, Phillips SF, et al. Eosinophilic gastroenteritis: a clinicopathological study of patients with disease of the mucosa, muscle layer, and subserosal tissues. Gut 1990:31:54-58.

9 Naylor AR. Eosinophilic gastroenteritis. Scott Med J 1990;35:163-5.

10 Khan S, Orenstein SR. Eosinophilic gastroenteritis. Pediatr Drugs 2002; 4:563-70

11 Lee CM, Changchien CS, Chen PC, et al. Eosinophilic gastroenteritis: 10 years experience. Am J Gastroenterol 1993:88:70-4.

12 Von Wattenwyl F, Zimmermann A, Netzer P. Synchronous first manifestation of an idiopathic eosinophilic gastroenteritis and bronchial asthma. Eur J Gastroenterol Hepatol 2001;13:721-5.

13 Shweiki E, West JC, Klena JW, et al. Eosinophilic gastroenteritis presenting as an obstructing cecal mass-a case report and review of the literature. Am J Gastroenterol 1999;94:3644-5.

14 Fenoglio LM, Benedetti V, Rossi C, et al. Eosinophilic gastroenteritis with ascites: a case report and review of the literature. Dig Dis $\mathrm{Sci}$ 2003;48:1013-20.

15 McNabb PC, Fleming CR, Higgins JA, et al. Transmural eosinophilic gastroenteritis with ascites. Mayo Clin Proc 1979;54:119-22.

16 Kristopaitis T, Neghme C, Yong SL, et al. Giant antral ulcer a rare presentation of eosinophilic gastroenteritis. Am J Gastroenterol 1997:92:1205-8.

17 Melamed I, Feanny SJ, Sherman PM, et al. Benefit of ketotifen in patients with eosinophilic gastroenteritis. Am J Med 1991;90:310-14.

18 Schwartz DA, Pardi DS, Murray JA. Use of montelukast as steroid-sparing agent for recurrent eosinophilic gastroenteritis. Dig Dis Sci 2001;46:1787-90.

19 Chen MJ, Chu CH, Lin SC, et al. Eosinophilic gastroenteritis: clinical experience with 15 patients. World J Gastroenterol 2003;9:2813-16. 